\title{
Identification of the Inhibitory Compounds for Metallo- $\beta$-lactamases and Structural Analysis of the Binding Modes
}

\author{
Taichi Kamo, ${ }^{a}$ Keiichi Kuroda, ${ }^{a}$ Shota Kondo, ${ }^{a}$ Usaki Hayashi, ${ }^{a}$ Satoshi Fudo, ${ }^{b}$ Tomoki Yoneda, ${ }^{c}$ \\ Akiko Takaya, ${ }^{a}$ Michiyoshi Nukaga, ${ }^{d}$ and Tyuji Hoshino*,a \\ ${ }^{a}$ Graduate School of Pharmaceutical Sciences, Chiba University; 1-8-1 Inohana, Chuo-ku, Chiba 260-8675, \\ Japan: ${ }^{b}$ HiLIFE (Institute of Biotechnology), University of Helsinki; Helsinki 00014, Finland: ${ }^{c}$ Division of Applied \\ Chemistry, Graduate School of Engineering, Hokkaido University; Kita 13, Nishi 8, Kita-ku, Sapporo 060-8628, \\ Japan: and ${ }^{d}$ Faculty of Pharmaceutical Sciences, Josai International University; 1 Gumyo, Togane, Chiba 283-8555, \\ Japan. \\ Received July 26, 2021; accepted September 23, 2021
}

Metallo- $\beta$-lactamases (MBLs) are significant threats to humans because they deteriorate many kinds of $\beta$-lactam antibiotics and are key enzymes responsible for multi-drug resistance of bacterial pathogens. As a result of in vitro screening, two compounds were identified as potent inhibitors of two kinds of MBLs: imipenemase (IMP-1) and New Delhi metallo- $\beta$-lactamase (NDM-1). The binding structure of one of the identified compounds was clarified by an X-ray crystal analysis in complex with IMP-1, in which two possible binding poses were observed. Molecular dynamics (MD) simulations were performed by building two calculation models from the respective binding poses. The compound was stably bound to the catalytic site during the simulation in one pose. The binding model between NDM-1 and the compound was constructed for MD simulation. Calculation results for NDM-1 were similar to those of IMP-1. The simulation suggested that the binding of the identified inhibitory compound was also durable in the catalytic site of NDM-1. The compound will be a sound basis for the development of the inhibitors for MBLs.

Key words metallo- $\beta$-lactamase; molecular dynamics simulation; thiazole; antimicrobial resistance; structural analysis; enzyme inhibitor

\section{Introduction}

Chemotherapy by antibiotics is the primary medical treatment for bacterial infectious diseases. To increase the therapeutic options, the development of novel medicines bearing the scaffold different from those of the currently approved drugs is strongly required. However, due to the difficulty in the discovery of novel classes of active chemicals and to the increasing diversity of resistant variants owing to over-use of the existing antibiotics, the rate of the new release of antimicrobial agents has decreased for the last four decades. ${ }^{1)}$

Beta-Lactamase is the key enzyme of the resistance for $\beta$-lactam antibiotics. Based on amino acid sequence homology, Ambler classified $\beta$-lactamases into four classes; A, B, C, and $\mathrm{D}^{2)}$ Class $\mathrm{A}, \mathrm{C}$, and $\mathrm{D} \beta$-lactamases have a serine residue at their catalytic site. In contrast, class $\mathrm{B} \beta$-lactamase holds one or two metal ions at the catalytic site and is named metallo- $\beta$ lactamase (MBL). The MBL-producing bacteria are resistant to broad kinds of $\beta$-lactam antibiotics, including carbapenems called "the last resort antibiotics." "Hence, MBLs are currently serious concerns for public health. ${ }^{4)}$ The major MBLs are Imipenemase (IMP) and New Delhi metallo- $\beta$-lactamase (NDM). IMP-1 was firstly detected in Japan in 1991, ${ }^{5)}$ and IMP-6, which had an amino acid sequence with only one residue difference from IMP-1, was also detected in Japan. ${ }^{6)}$ In 2007, NDM-1 was isolated from a patient from India. ${ }^{7)}$ In spite of many efforts to develop inhibitors for MBLs, ${ }^{8-10)}$ the compound potency or the chemical property is still insufficient for clinical use. Therefore, the discovery of a promising chemical scaffold is demanded at present. In this study, we performed an in vitro screening with a collection of the synthesized com- pounds targeting the metal-containing enzymes. The inhibitory activities were measured both for IMP-1 and NDM-1, and two potent inhibitory compounds were identified. The binding structure of one of the identified compounds to MBL was analyzed by X-ray crystallography. Molecular dynamics (MD) simulations, major calculation methods in computational biology, ${ }^{11)}$ were carried out to evaluate the stability of the binding poses observed in the crystal structures.

\section{Results and Discussion}

Screening Assay To find the inhibitory compounds for the enzymatic activity of MBLs, the inhibition rate was measured for about 600 in-house compounds. Most of the compounds were synthesized through the research of developing the inhibitors targeting the ribonuclease (RNase) $\mathrm{H}$ activity of human immunodeficiency virus type 1 (HIV-1) reverse transcriptase $^{12,13)}$ or the endonuclease activity of influenza virus polymerase. ${ }^{14-16)}$ As with the MBLs, both HIV-1 RNase $\mathrm{H}^{17,18)}$ and influenza endonuclease ${ }^{19-21)}$ activities are functionalized by two divalent metal ions located at their catalytic sites. Therefore, our chemical collection was anticipated to contain potent compounds blocking the enzymatic activity of MBLs. In the screening, 47 compounds were selected by measuring the inhibition rate at a concentration of $100 \mu \mathrm{M}$, followed by 12 compounds which one was developed as the tyrosine kinase receptor $\mathrm{B}(\mathrm{TrkB})$ inhibitor $^{22,23)}$ by measuring the inhibition rate at a concentration of $20 \mu \mathrm{M}$. Then, all 12 compounds were tested at 4.0 and $0.8 \mu \mathrm{M}$, two compounds $\mathbf{1}$ and $\mathbf{2}$ were identified to be potential inhibitors (Figs. 1a, 1b). Cephalosporin $\mathrm{C}$ was used as an antibacterial agent. Compound $\mathbf{1}$ had a 
(a)<smiles>O=C1CCc2cc(-c3csc(COc4ccccc4)n3)ccc2N1</smiles>

(b)<smiles>COC(=O)c1ccc(OCc2cccc(O)c2)c(OC)c1</smiles>

Fig. 1. (a, b) Chemical Structures of the Compounds Identified as Inhibitors for the Hydrolysis Activity of MBLs
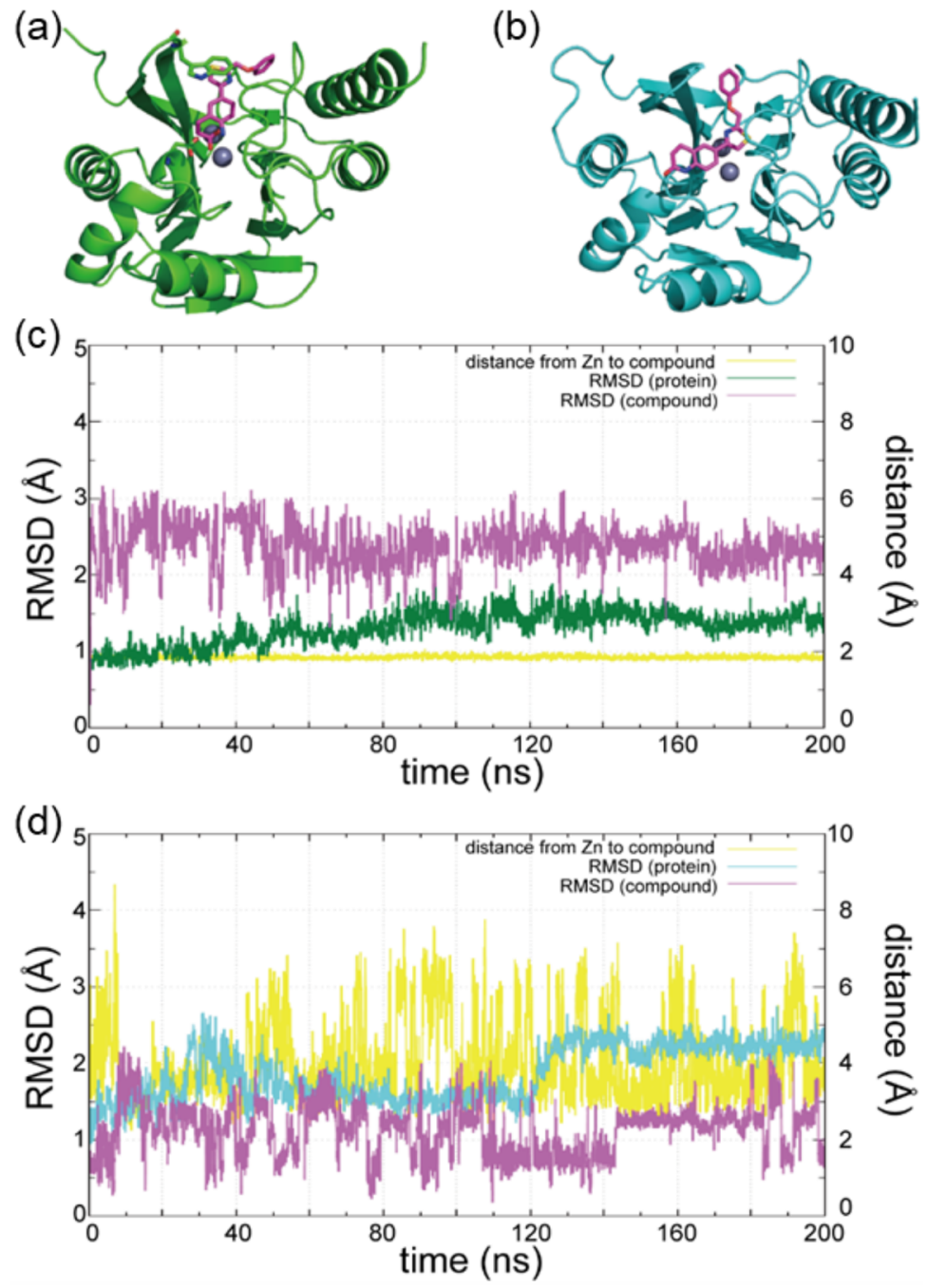

Fig. 2. (a) Binding Pose A of the IMP-1 and Compound 1 Complex

(b) Binding pose B of the IMP-1 and compound 1 complex. (c) Change in the root-mean-square deviation (RMSD) and change in the closest distance from compound 1 to IMP-1 during the simulation for pose A. Green and magenta represent the RMSDs for protein and compound 1, respectively. The closest distance is represented by yellow. (d) Changes in RMSD and the closest distance along with the simulation for pose B. (Color figure can be accessed in the online version.)

strong UV absorption peak at $301 \mathrm{~nm}$ owing to the presence of a $o$-phenylene-fused $\delta$-lactam ring $(3,4$-dihydro- $2(1 H)$ quinolinone), and the UV-light at $260 \mathrm{~nm}$ was adsorbed both by cephalosporin $\mathrm{C}$ and compound $\mathbf{1}$. Hence, the inhibitory rate did not reach 1.0, even at high compound concentrations (Supplementary Figs. S1b, S1c).

Evaluation of Inhibitory Activity $\mathrm{The}^{\mathrm{IC}_{50}}$ values both for NDM-1 and IMP-1 were determined for the two identified compounds by measuring the inhibition rate in duplicate with several different concentrations of the compounds (Supplementary Figs. S1b, S1c, S1e, S1f). The $\mathrm{IC}_{50}$ value of compound $\mathbf{1}$ was lower than that of compound 2 for IMP-1. The inhibitory activity was also observed for NDM-1 both for the compounds.

Crystallography A single crystal of IMP-1 was obtained in a complex form with compound 1 (PDB\#; 6JKA). An X-ray crystal analysis showed that four IMP-1 molecules were packed in an asymmetric unit cell (Supplementary Fig. S3a). Three compound $\mathbf{1}$ molecules were observed in the unit cell. Two of the three were located at the catalytic site of the enzyme. These two binding structures were labeled as pose A and pose $\mathrm{B}$. The other one was observed at the space between two IMP-1 molecules, and its location was far apart from the 
(a)

(b)
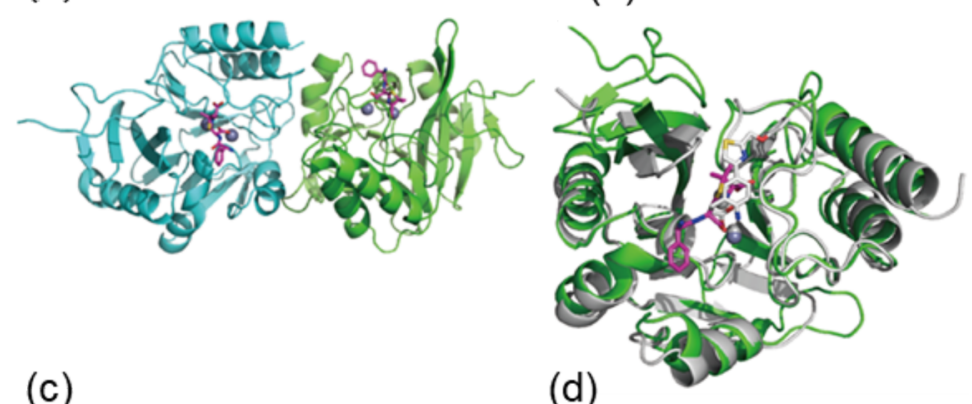

(c)

(d)
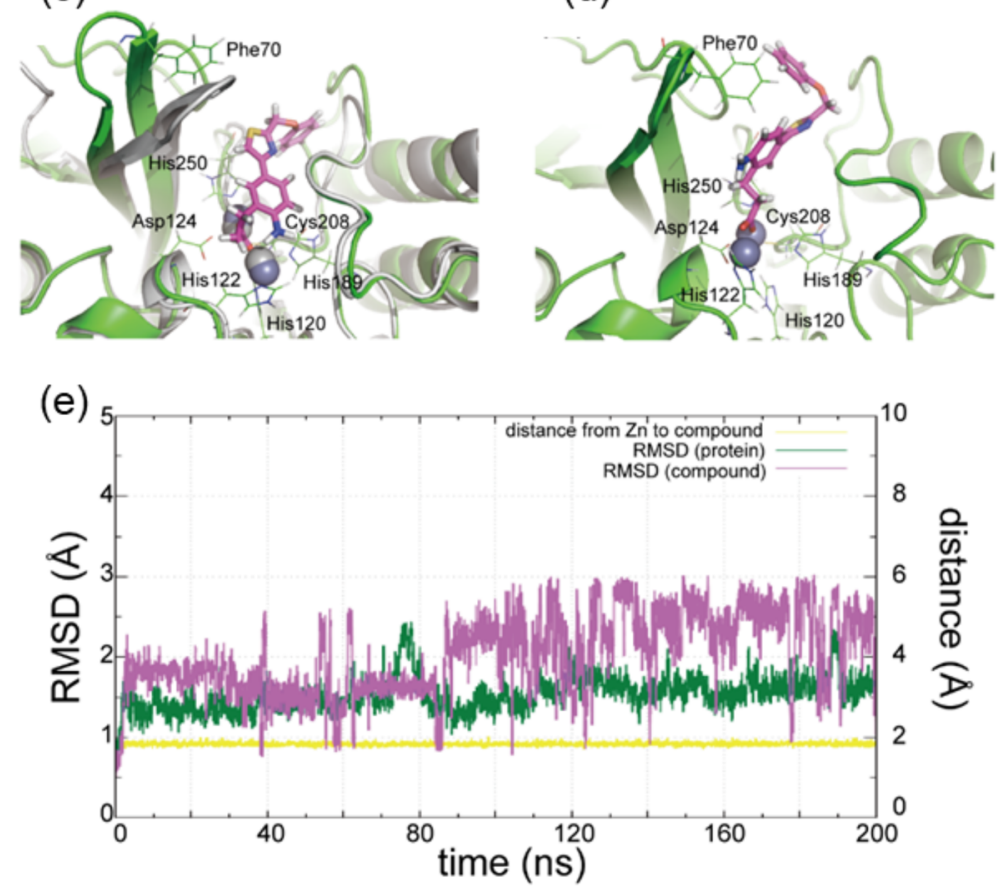

Fig. 3. (a) Crystal Structure of the Complex of NDM-1 and Ampicillin (PDB\#; 6JKB)

(b) Superposition of crystal structure of the ampicillin-bound NDM-1 (green) onto that of compound 1-bound IMP-1 (grey) in pose A. (c) Magnified view of (b). The ampicillin molecule is omitted. (d) Representative structure of the simulation trajectory for the last $20 \mathrm{~ns}$ in the complex of compound 1 and NDM-1. (e) Changes in RMSD and the closest distance from compound $\mathbf{1}$ to NDM-1 along with the simulation time. (Color figure can be accessed in the online version.)

catalytic site. Hence, either of the binding poses A and B was judged to be closely involved in the inhibition of IMP-1. In pose A (Fig. 2a), the $\delta$-lactam ring of compound $\mathbf{1}$ was hydrolyzed, and the generated carboxy group formed the coordinate bonds to $\mathrm{Zn}^{2+}$ ions. The positions of the functional groups of compound 1 were assigned both by the Fo-Fc omit map (Fig. $3 b$ ) and the 2Fo-Fc density map (Supplementary Figs. S3c, S3d). The thiazole ring made a $\pi-\pi$ interaction with $\operatorname{Trp} 67$, which covered compound $\mathbf{1}$ from the opposite side to $\mathrm{Zn}^{2+}$ ions at the catalytic pocket. In pose B (Fig. 2b), the thiazole ring was coordinated to $\mathrm{Zn}^{2+}$ ions, and the $\delta$-lactam ring was not hydrolyzed. The omit map (Supplementary Fig. S3e) and the density map (Supplementary Figs. S3f, S3g) suggested that the carbonyl group of the $\delta$-lactam ring formed a hydrogen bond with Ser119 and that Trp67 made a $\pi-\pi$ interaction with the phenyl group at the terminal moiety.

Molecular Dynamics Simulation To examine the stability of the binding structures of poses A and B, 200ns MD simulations were performed, in which the calculation models were built from the respective crystal structures. The force field parameters were ff $14 \mathrm{SB}$ for proteins ${ }^{24}$ and gaff 2 for the compound. ${ }^{25)}$ Atom charges of the compound were determined by the quantum chemical calculation in a similar manner to the previous works. ${ }^{26-28)}$ Compound $\mathbf{1}$ stayed at the catalytic site all through the simulations both for poses A and B. The binding structures were, however, changed from those of the starting models. The deviation from the starting structure was remarkable for pose $\mathrm{B}$, compared to that for pose $\mathrm{A}$. The stability of the binding pose A was further confirmed from the low fluctuation in the minimum distance between compound 1 and $\mathrm{Zn}^{2+}$ ions. The ceaseless changes in the interactions at pose B suggested the low stability of pose B (Fig. 2d). The large root-mean-square deviation (RMSD) and compact principal component analysis (PCA) of pose B suggested that the alteration of the binding pose of $\mathrm{B}$ was primarily due to the positional displacement of compound $\mathbf{1}$ instead of conformational change. From these simulation results, it was concluded that pose A was a stable binding structure of compound $\mathbf{1}$ to IMP-1 but pose B was not so stable.

The protein crystal of NDM-1 was obtained when ampicillin was included in the crystallization droplet. A complex structure of NDM-1 and ampicillin was observed in the X-ray structure analysis (Fig. 3a). The crystal structure of IMP-1 and NDM-1 were highly compatible with each other, and the posi- 
tions of the two $\mathrm{Zn}^{2+}$ ions were consistent between the two enzymes (Fig. 3b, Supplementary Fig. S9). The atom coordinates of compound $\mathbf{1}$ were extracted from the IMP-1-compound $\mathbf{1}$ complex and placed on the NDM-1 crystal structure (Figs. 3a, $3 \mathrm{~b}$ ). Using the two hypothetical binding poses of compound $\mathbf{1}$ and NDM-1, MD simulations were performed to examine their stability. The simulation suggested that compound $\mathbf{1}$ interacted more stably with NDM-1 in pose A, similar to the results of those of IMP-1 (Figs. 3d, 3e). From these results of simulation, compound 1 was expected to inhibit the enzymatic activity of both IMP-1 and NDM-1. In the metal enzymes, for instance like carbonyl anhydrate II, amino acid residues with a carboxy group at their side chain are responsible for holding the metals at the catalytic site. ${ }^{29)}$ Hence, it is natural that the inhibitory compounds for MBLs contain a carboxy group to attach themselves to the di-zinc center stably. In the present work, the $\delta$-lactam ring of compound $\mathbf{1}$ was hydrolyzed to generate a carboxy group, which resulted in the association of the compound to the catalytic center of IMP-1 and NDM-1.

Structural Modification of Compound 1 According to the structural analysis on the complex of MBLs and compound 1 (Figs. 2, 3, S3), the phenyl group at the opposite side to the $\delta$-lactam ring formed a $\mathrm{CH}-\pi$ or $\pi-\pi$ interaction with Tyr67 of IMP-1 or Phe70 of NDM-1. Since the terminal phenyl group occasionally changed its position during MD simulation, the chemical modification of the phenyl group may increase the inhibitory activity against MLBs due to the improvement of the structural complementarity with the proteins. Hence, several compounds, phenyl group of which was modified by adding a chemical substituent, were synthesized. The inhibitory activity against IMP-1 was measured for the synthesized compounds (Table 1), and their synthetic routes were shown in Description S1. The conversion of hydrogen atom at the para-position into methyl group showed a 3-fold increase of the compound potency (compound 3), while that into fluorine largely decreased the activity (compound 4). These changes in inhibitory activity suggested that the phenyl group had a significant $\mathrm{CH}-\pi$ or $\pi-\pi$ interaction with protein residues. The conversion of the hydrogen into a methoxy group resulted in the complete loss of the inhibitory activity in spite that the methoxy group connected to an aromatic ring usually works as an electron donor (compound 5). Hence, the size of the functional group that can be adapted at the phenyl para-position is limited. When both electron-donating and -withdrawing substituents were added to the phenyl (com-

Table 1. Inhibition Concentrations $\left(\mathrm{IC}_{50}\right)$ of the Derivative Compounds for IMP-1

\begin{tabular}{cccc}
\hline & $\mathrm{H}$ & $\mathrm{H}$ & $\mathrm{IC}_{50}(\mu \mathrm{M})$ \\
\hline $\mathbf{1}$ & $\mathrm{Re}$ & $\mathrm{H}$ & 27.3 \\
$\mathbf{3}$ & $\mathrm{F}$ & $\mathrm{H}$ & $>100$ \\
$\mathbf{4}$ & $\mathrm{OMe}$ & $\mathrm{H}$ & $>100$ \\
$\mathbf{5}$ & $\mathrm{Me}$ & $\mathrm{Cl}$ & 36.9 \\
$\mathbf{6}$ & $\mathrm{Me}$ & $\mathrm{Me}$ & 1.52 \\
$\mathbf{7}$ & $\mathrm{Me}$ &
\end{tabular}

pound 6), the inhibitory activity was comparable to the original one. In contrast, when two electron-donating substituents were added to the para- and ortho-positions (compound 7), the compound potency was much promoted. The $\mathrm{IC}_{50}$ value of the two methyls-attached compound was $1.5 \mu \mathrm{M}$, which was an 18 -fold increase of the inhibitory activity.

\section{Conclusion}

Two compounds to inhibit the enzymatic activity of MBLs, IMP-1 and NDM-1, were identified by an in vitro screening with 600 chemicals. The $\mathrm{IC}_{50}$ value of one of the identified compounds was $27 \mu \mathrm{M}$ for IMP-1. An X-ray crystal analysis was carried out to clarify the binding structure of the compound and IMP-1. In the crystal structure, two binding poses were observed for the compound at the catalytic site of IMP-1. To examine the stability of the binding poses, $200 \mathrm{~ns}$ MD simulations were performed with two kinds of calculation models built from the respective binding poses. The compound stably stayed at the catalytic site during the simulation in one binding pose. MD simulation was also performed for $200 \mathrm{~ns}$ with a calculation model built from the ampicillin-replaced structure, which resulted in the stable binding of the compound $\mathbf{1}$ at the catalytic site of NDM-1. Hence, it is concluded that the compound is a sound basis for the development of the inhibitors for MBLs.

\section{Experimental}

Details of experimental methods are described in supplementary materials.

Acknowledgments Calculations were performed at the Research Center for Computational Science, Okazaki, Japan. $\mathrm{X}$-ray diffractions were acquired at the Photon Factory, Tsukuba, Japan (proposal No. 2018G613, 2020G590). A part of this work was supported by a grant for Scientific Research C from the Japan Society for the Promotion of Science (21K07050).

Conflict of Interest The authors declare no conflict of interest.

Supplementary Materials The online version of this article contains supplementary materials.

\section{References}

1) Spellberg B., Guidos R., Gilbert D., Bradley J., Boucher H. W., Scheld W. M., Bartlett J. G., Edwards J. Jr., Clin. Infect. Dis., 46, 155-164 (2008)

2) Drawz S. M., Bonomo R. A., Clin. Microbiol. Rev., 23, 160-201 (2010).

3) El-Gamal M. I., Brahim I., Hisham N., Aladdin R., Mohammed H., Bahaaeldin A., Eur. J. Med. Chem., 131, 185-195 (2017).

4) Tooke C. L., Hinchliffe P., Bragginton E. C., Colenso C. K., Hirvonen V. H. A., Takebayashi Y., Spencer J., J. Mol. Biol., 431, 3472-3500 (2019)

5) Osano E., Arakawa Y., Wacharotayankun R., Ohta M., Horii T., Ito H., Yoshimura F., Kato N., Antimicrob. Agents Chemother., 38, 71-78 (1994).

6) Yano H., Kuga A., Okamoto R., Kitasato H., Kobayashi T., Inoue M., Antimicrob. Agents Chemother., 45, 1343-1348 (2001).

7) Yong D., Toleman M. A., Giske C. G., Cho H. S., Sundman K., Lee K., Walsh T. R., Antimicrob. Agents Chemother., 53, 5046-5054 (2009). 
8) Linciano P., Cendron L., Gianquinto E., Spyrakis F., Tondi D., ACS Infect. Dis., 5, 9-34 (2019).

9) Wang T., Xu K., Zhao L., Tong R., Xiong L., Shi J., Eur. J. Med. Chem., 223, 113667 (2021).

10) Zhang Y. J., Liu X. L., Wang W. M., Chen C., Zhao M. H., Yang K. W., Chem. Pharm. Bull., 67, 135-142 (2019).

11) Mahmood I., Liu X., Neya S., Hoshino T., Chem. Pharm. Bull., 61, 426-437 (2013).

12) Yanagita H., Urano E., Matsumoto K., Ichikawa R., Takaesu Y., Ogata M., Murakami T., Wu H., Chiba J., Komano J., Hoshino T., Bioorg. Med. Chem., 19, 816-825 (2011).

13) Yanagita H., Fudo S., Urano E., Ichikawa R., Ogata M., Yokota M., Murakami T., Wu H. G., Chiba J., Komano J., Hoshino T., Chem. Pharm. Bull., 60, 764-771 (2012).

14) Fudo S., Yamamoto N., Nukaga M., Odagiri T., Tashiro M., Neya S., Hoshino T., Bioorg. Med. Chem., 23, 5466-5475 (2015).

15) Fudo S., Yamamoto N., Nukaga M., Odagiri T., Tashiro M., Hoshino T., Biochemistry, 55, 2646-2660 (2016).

16) Fudo S., Qi F., Nukaga M., Hoshino T., Cryst. Growth Des., 17, 534-542 (2017).

17) Davies J. F. 2nd, Hostomska Z., Hostomsky Z., Jordan S. R., Matthews D. A., Science, 252, 88-95 (1991).

18) Katayanagi K., Miyagawa M., Matsushima M., Ishikawa M. Kanaya S., Nakamura H., Ikehara M., Matsuzaki T., Morikawa K., J. Mol. Biol., 223, 1029-1052 (1992).

19) Yuan P., Bartlam M., Lou Z., Chen S., Zhou J., He X., Lv Z., Ge
R., Li X., Deng T., Fodor E., Rao Z., Liu Y., Nature (London), 458, 909-913 (2009)

20) Dias A., Bouvier D., Crépin T., McCarthy A. A., Hart D. J., Baudin F., Cusagck S., Ruigrok R. W. H., Nature (London), 458, 914-918 (2009).

21) Zhao C., Lou Z., Guo Y., Ma M., Chen Y., Liang S., Zhang L., Chen S., Li X., Liu Y., Bartlam M., Rao Z., J. Virol., 83, 9024-9030 (2009).

22) Nakamura Y., Suganami A., Fukuda M., Hasan M. K., Yokochi T., Takatori A., Satoh S., Hoshino T., Tamura Y., Nakagawara A., Cancer Med., 3, 25-35 (2014).

23) Fukuda M., Takatori A., Nakamura Y., Suganami A., Hoshino T., Tamura Y., Nakagawara A., Neurochem. Int., 97, 42-48 (2016).

24) Maier J. A., Martinez C., Kasavajhala K., Wickstrom L., Hauser K. E., Simmerling C., J. Chem. Theory Comput., 11, 3696-3713 (2015).

25) Wang J., Wolf R. M., Caldwell J. W., Kollman P. A., Case D. A., J. Comput. Chem., 25, 1157-1174 (2004).

26) Qi F., Fudo S., Neya S., Hoshino T., Chem. Pharm. Bull., 62, 568577 (2014).

27) Qi F., Fudo S., Neya S., Hoshino T., J. Chem. Inf. Model., 55, 1673-1685 (2015).

28) Mahmood I., Liu X., Neya S., Hoshino T., Chem. Pharm. Bull., 61, 426-437 (2013).

29) Kitahara M., Fudo S., Yoneda T., Nukaga M., Hoshino T., Cryst. Growth Des., 19, 6004-6010 (2019). 